\title{
Lenalidomide in 5q minus myelodysplastic syndrome: how long is enough?
}

\author{
Donat Dürr • Raffaele Daniele Siciliano • \\ Yvonne Hummel • Alix O'Meara • Anita Hirschi • \\ Roger Burkhard $\cdot$ Hanspeter Honegger
}

Received: 7 June 2009 /Accepted: 14 June 2009 / Published online: 27 June 2009

(C) Springer-Verlag 2009

\section{Dear Editor,}

In the spring of 2005, a female patient aged 67 years at that time was referred to our service because of progressive haemoglobin decline. There was no history of bleeding. In 1997, in an external institution, bone marrow aspiration and cytogenetic analysis yielded the diagnosis of a myelodysplatic syndrome with a $5 \mathrm{q}-$-minus deletion (del5q), type 5qminus syndrome (5q-Syn). Our laboratory tests revealed severe anaemia (haemoglobin $5 \mathrm{~g} / \mathrm{dL}$ ) as well as leucopenia $\left[2.8 \times 10^{9} / \mathrm{L}\right.$ (normal range, 3.7-9.6 $\times 10^{9} / \mathrm{L}$ )], while overall neutrophil and thrombocyte counts were in the normal ranges. Given the symptomatic anaemia, erythrocyte concentrate transfusions were initiated. In May 2006, we started oral therapy with lenalidomide $(10 \mathrm{mg}$ /day for 21 days; 28-day cycle). Already by mid-July 2006, the patient had reached a haemoglobin value of $12 \mathrm{~g} / \mathrm{dL}$ (Fig. 1). Until October 2006, four lenalidomide cycles were administered in total. Except for transient neutropenia grade III, no other side effects were recorded. Follow-up bone marrow examination in November 2006 revealed haematological and a borderline cytogenetic remission ("borderline" because only 18 metaphases were analysed and not 20 as postulated by List [1]). Lenalidomide therapy was not restarted. In further bone marrow examinations, the reappearance of the del5q (March 2007, 18/79 metaphases (23\%); March 2008, 8/20 (40\%) metaphases) was detected. Nevertheless, 26 months after treatment cessation, there is

D. Dürr $(\varangle) \cdot$ R. D. Siciliano $\cdot$ Y. Hummel $\cdot$ A. O'Meara

A. Hirschi $\cdot$ R. Burkhard $\cdot$ H. Honegger

Medical Oncology, Stadtspital Triemli,

Birmensdorferstrasse 497,

CH-8063 Zurich, Switzerland

e-mail: donat.duerr@triemli.stzh.ch still no evidence of anaemia or cytopenia in peripheral blood and no change in mean cellular volume.

This is - to our knowledge - the first long-term follow-up of a patient with a 5q-Syn having achieved haematological and borderline cytogenetic remission under lenalidomide, who then ceased therapy. In few other cases published, lenalidomide was stopped because of drug intolerance and before the patients achieved haematological remission [2]. In our case, lenalidomide was paused because of beginning polyglobulia - a well-known adverse effect of lenalidomide [3]. Furthermore, the current expert recommendations to continue treatment after achieving haematological remission [4] did not exist yet.

The therapeutic mechanism of action of lenalidomide is matter of speculation. It has been suggested that lenalidomide exerts a del5q cytotoxic effect $[1,5]$ and an immunomodulating effect, which could have a positive effect on bone marrow microenvironment and therefore on haematopoiesis $[2,6]$. Accounting for these possible mechanisms of action, the direct cytotoxic effect of lenalidomide gives us an explanation for the rapid reappearance of del5q clone. Treatment discontinuation with achievement of borderline cytogenetic remission appears to be too short for long-term suppression of the del5q clone, probably because of the incomplete elimination of CD34 del5q-progenitor cells, insofar as complete elimination is possible [7]. However, it is noteworthy that our patient has not suffered haematological relapse for more than 26 months since discontinuing treatment, despite the rapid reappearance of the 5q-minus clone. We believe that the microenvironmental effect of lenalidomide may prolong the time interval between cytogenetic and haematologic relapse in 5q-Syn, at least for some patients. This phenomenon would be an argument against a continuous 
Fig. 1 Complete haematological remission was still evident 26 months after lenalidomide discontinuation

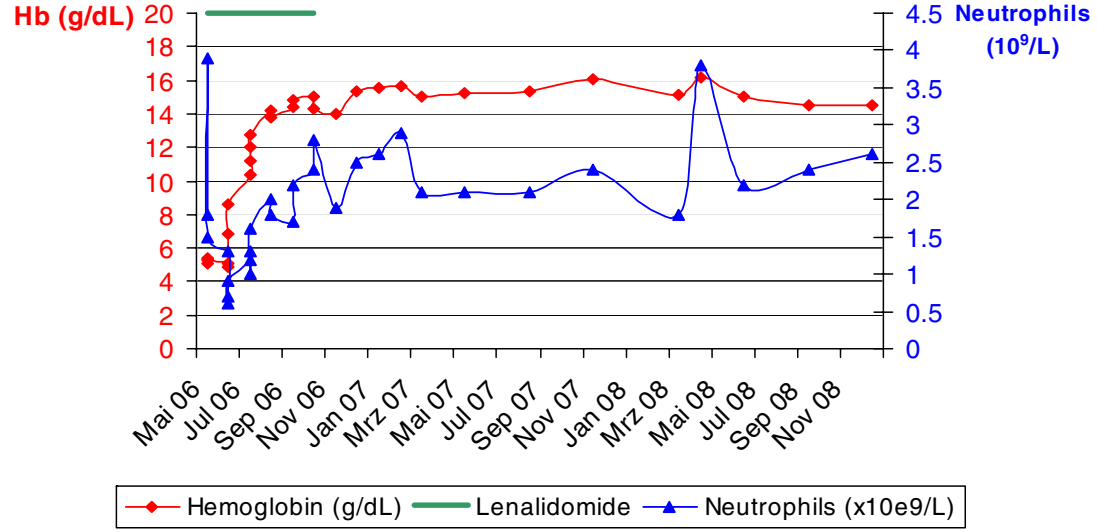

lenalidomide therapy, which is associated with possible adverse effects and enormous costs.

\section{Glossary of abbreviations}

Granulo Granulocytes

$\mathrm{Hb} \quad$ Haemoglobin

del5q 5q-minus deletion

5q-Syn 5q-minus syndrome

\section{References}

1. List A, Kurtin S, Roe DJ, Buresh A, Mahadevan D, Fuchs D et al (2005) Efficacy of lenalidomide in myelodysplastic syndrome. N Engl J Med 352(6):549-557

2. Giagounidis A, Haase S, Heinsch M, Göhring G, Schlegelberger B, Aul C (2007) Lenalidomide in the context of complex karyotype of interrupted treatment: case reviews of $\operatorname{del}(5 q)$ MDS patients with unexpected responses. Ann Hematol 86:133-137

3. Kelaidi C, Eclache V, Fenaux P (2008) The role of lenalidomide in the management of myelodysplasia with del 5q. Br J Hematol 140 (3):267-278

4. Giagounidis A, Fenaux P, Mufti GJ, Muus P, Platzbecker U, Sanz $G$ et al (2008) Practical recommendations on the use of lenalidomide in the management of myelodysplastic syndromes. Ann Hematol 87:345-352

5. List A, Dewald G, Bennett J, Giagounidis A, Raza A, Feldman E et al (2006) Lenalidomide in the myelodysplastic syndrome with chromosome 5q deletion. N Engl J Med 355(14):1456-1465

6. Raza A, Reeves JA, Feldman EJ, Dewald GW, Bennett JM, Deeg HJ et al (2008) Phase 2 study of lenalidomide in transfusiondependent, low-risk, and intermediate-1-risk myelodysplastic syndromes with karyotypes other than deletion 5q. Blood 111:86-93

7. Mohr B, Oelschlaegel U, Thiede C, Stewart MM, Ehninger G, Platzbecker U (2009) The response to lenalidomide of myelodysplastic syndrome patients with deletion del $(5 \mathrm{q})$ can be sequentially monitored in CD34+ progentior cells. Haematologica 94:430-431. doi:10.3324/haematol13865 